\title{
28 Research Square \\ Incidence and Risk Factors of postoperative \\ Dysphagia following Cervical Spine Surgery
}

Jie Yu

Beijing Jishuitan Hospital

Xiaohui Tao ( $\sim$ 412208939@qq.com )

Beijing Jishuitan Hospital https://orcid.org/0000-0002-5683-5678

Research article

Keywords: dysphagia, cervical spine surgery, anterior cervical discectomy and fusion, laminoplasty, neck collar

Posted Date: August 21st, 2020

DOI: https://doi.org/10.21203/rs.3.rs-46131/v1

License: (1) This work is licensed under a Creative Commons Attribution 4.0 International License. Read Full License 


\section{Abstract}

Background. Dysphagia is a known complication of anterior cervical spine surgery and may be prolonged or occasionally serious. However, the association between posterior cervical spine surgery and the development of dysphagia is rare discussed. Furthermore, the risk factors of postoperative dysphagia are not well understood.

Methods. Three hundred and fifty four patients were reviewed in this study, including 172 patients undergoing AC procedure, and 182 patients with PC procedure between June 2007 and May 2012. All patients were given the same postoperative management. The presence and severity of dysphagia were reported during the telephone interviews performed at $1,3,6$, and 12 months after the procedure. The incidence and risk factors of dysphagia after cervical spine surgery were studied.

Results. Of all 354 patients, $12.8 \%$ AC and $9.4 \%$ PC patients reported dysphagia at early postoperative assessment. The overall incidence for dysphagia at $1,3,6$ and 12 months were decreased to $9.3 \%, 7.0 \%$, $4.6 \%$ and $3.5 \%$ in AC group, and $6.0 \%, 4.4 \%, 2.2 \%$ and $1.6 \%$ in PC group, respectively. Long term use of Neck Collar after surgery may lead to postoperative dysphagia. Other risk factors such as age, gender, $\mathrm{BMI}$, operative time, blood loss, procedure type, revision surgery and operative levels did not significantly influence the incidence of postoperative dysphagia.

Conclusions. Postoperative dysphagia is common after anterior and posterior cervical spine surgery. The incidence and severity of dysphagia both decrease over time. Long term use of neck collar after surgery is related of postoperative dysphagia. The neck collar should be worn no longer than four weeks after surgery. Age, gender, BMl, operative time, blood loss, procedure type, revision surgery and operative levels are not risk factors of postoperative dysphagia.

\section{Background}

Anterior cervical decompression and discectomy, plate and screw instrumentation, bone graft and fusion, (referred to as ACDF, Anterior cervical discectomy and fusion) was the most common procedure for cervical spondylosis. This proved to be effective and safe in clinical practice. On the other hand, patients undergoing ACDF surgery presented with postoperative dysphagia, which was one of the most frequent complications according to the literature. The occurrence of postoperative dysphagia in ACDF group varied differently from $4 \%-71 \%^{1-25}$, but there were rare cases of severe dysphagia. Meanwhile, a portion of patients undergoing posterior cervical spinous process-splitting laminoplasty (PC procedure) also develop dysphagia after surgery, which has rarely been mentioned in previous research.

Domestic studies concerning the mechanisms of postoperative dysphagia development in short- and long-term outcomes after cervical spine surgery were relatively few. Many potential risk factors have been reported, including age ${ }^{1,2}$, gender ${ }^{3,4}$, intraoperative esophagus traction ${ }^{5,6}$, long segmental surgery 7,8 , postoperative persistent pain ${ }^{9,10}$, prolonged procedure time ${ }^{11}$, high surgical position ${ }^{12}$, revision surgery ${ }^{13}$, 
esophageal intubation ${ }^{14}$, and massive blood loss ${ }^{15}$. In addition, other risk factors were associated with dysphagia development such as thickness of internal plate ${ }^{16}$, intraoperative injury of esophagus ${ }^{17}$, postoperative cervical hematoma formation ${ }^{18}$, the use of BMP- $2^{19}$, postoperative soft swelling ${ }^{20}$, scar formation and tissue adhesion ${ }^{21}$, internal compression of the esophagus ${ }^{22}$ and recurrent laryngeal nerve injury ${ }^{23-25}$.

Meanwhile, to access whether other confounding factors affected the occurrence of dysphagia, we collected clinical and operative characteristics. Logistic regression was used in analyzing these clinical data, including gender, age, BMI, operation time, blood loss, usage time of neck collar, type of surgical procedure (anterior/posterior), revision surgery, number of surgical segments, the superior segment of surgical cite, whether the third cervical vertebrae was included.

\section{Methods}

\section{Patient Selection}

From June 2007 to May 2012, a total of 198 patients were treated with anterior cervical decompression and plate fixation. 12 months follow-up was completed in 172 patients. There were 118 males and 54 females with a mean age of 48.8 (range 18-72) years. There were 102 patients diagnosed as cervical spondylotic myelopathy, 52 patients as cervical spondylotic radiculopathy, 10 patients as mixed type spondylosis, and 8 patients as cervical spinal fracture. Within the same session, a total of 220 patients underwent spinous process-splitting laminoplasty and at least 1 year follow-up was completed in 182 patients. 137 patients were male and 45 female with a mean age of 55.9 (range 25-76) years. There were 108 patients diagnosed as cervical spondylotic myelopathy, 54 patients as cervical OPLL, and 20 patients as cervical spinal canal stenosis.

The inclusion criteria was as follows: i) No preoperative dysphagia symptom; ii) No diagnosis of cervical neoplasm; iii) Preoperative and postoperative Japanese Orthopedic Association (JOA) scores were accessible; iv) Preoperative (obtained within 3 months) and postoperative (obtained within 3 months) cervical X-rays were accessible; v) Patients could make routine follow up by telephone interview; vi) Details of surgical procedures were reported in full medical records; vii) No diagnosis of schizophrenia or neurotic symptoms; viii) No diagnosis of oral diseases after surgery.

The exclusion criteria were listed as following: i) Patients could not make routine follow up; ii) Preoperative and Postoperative JOA scores, general information data, cervical X-rays were missing; iii) Patients presented with dysphagia before surgery, including 3 of 198 patients in the ACDF group and 4 of 152 patients in the CDR group.

\section{Surgical Protocols}

AC procedure. 
The AC procedure was performed via the standard Smith-Robinson approach to the cervical spine under general anesthesia and endotracheal intubation. A right-sided approach was typically performed if the surgical fields were above the $\mathrm{C} 6$ level; under $\mathrm{C} 6$ level we would like to choose the left-sided approach to decrease the potential injury to the right recurrent laryngeal nerve. C-arm fluoroscopy was used to mark an appropriate skin incision. Blunt dissection was used to expose the anterior aspect of the cervical spine. Deep retraction of the soft tissue was achieved with smooth-edged self-retaining retractors. Direct decompression is performed by removing the offending discs and osteophytes on the preoperative radiographs. Bone fusion was obtained using either autologous iliac bone graft or coral bone allograft. Anterior cervical plating with locking screws was used in all cases involving instrumentation (Fig. 2.a). All patients were asked to use a firm cervical collar for at least 2 weeks postoperatively.

PC procedure.

The patients of PC group were treated with spinous process-splitting laminoplasty, as first described by Kurokawa in 1982 and improved by us during recent years. Like AC procedure, patients were under general anaesthesia and endotracheal intubation. A midline linear incision was performed, and soft tissues were divided to expose the posterior elements. The dorsal part of each spinous process was removed. Lateral gutters for the hinge were created using a diamond burr. The lamina was then cut at the midline by using a threadwire-saw instead of diamond burr, which markedly shortened the mean time of surgery and lessened the mean blood loss. Coral bone grafts were shaped to fit the spaces and secured with wire. As a general rule, C4-C6 laminoplasty was performed to provide extensive decompression while conserving the $\mathrm{C} 3$ and $\mathrm{C} 7$ spinous process (Fig. 2.b), which was different from Kurokawa's method. As an exception, C4-C7 laminoplasty was performed for $\mathrm{C} 6 / 7$ disc herniation or stenosis. At least Two weeks of neck immobilization with a collar after surgery were recommended to these patients.

\section{Data Collection}

The presence and severity of dysphagia were recorded at early postoperative, 1, 3, 6 and 12 months after the procedure, by face-to-face questioning or telephone interview. The dysphagia grading system defined by Bazaz et al ${ }^{1}$ was used (Table 1 ).

Table 1

Bazaz grading system for dysphagia

\begin{tabular}{|lll|}
\hline Severity & Liquid & Solid \\
\hline None & None & None \\
Mild & None & Rare \\
Moderate & None or Rare & Occasional (only with specific foods) \\
Severe & Present & Frequent (majority of Solids) \\
\hline
\end{tabular}

This system includes four grades: none, mild, moderate, or severe dysphagia based on the subjective symptoms. 


\section{Statistical Analysis:}

Comparison of the overall incidence of dysphagia between the AC and PC group early preoperatively and at each time period after surgery $(1,3,6$, and 12 months) was performed according to the results of telephone interview. A Logistic regression analysis was also used to investigate the association of dysphasia with several documented risk factors, with dysphasia as the dependent variable. The independent variables included age, gender, BMI, blood loss, length of surgery, Usage time of neck collar after operation, primary or revision, AC or PC procedure (procedure type), and operative levels. All reported $p$ values are two-tailed, and the level of statistical significance was $p<0.05$. All statistical analysis was performed using SPSS, version 19.0(SPSS Inc., Chicago, IL, USA).

\section{Results:}

For AC group, the prevalence and severity of dysphagia at early postoperative was $12.8 \%$ (Fig. 1) (mild: $5.8 \%$, moderate: $4.1 \%$, and severe: $2.9 \%$ ) and decreased over time to $9.3 \%$ at 1 month (mild: $4.1 \%$, moderate: $2.3 \%$, and severe: $2.9 \%$ ), $7.0 \%$ at 3 months (mild: $2.3 \%$, moderate: $1.7 \%$, and severe: $2.9 \%$ ), $4.7 \%$ at 6 months (mild: $0.6 \%$, moderate: $1.7 \%$, and severe, $2.3 \%$ ), and $3.5 \%$ over 12 months (mild: $0.6 \%$, moderate: $1.7 \%$, and severe: $1.2 \%$ )(Table 2). As for patients of PC group, subjective complain of developing dysphagia at early postoperative was $9.4 \%$ (Fig. 1) (mild: $5.5 \%$, moderate: $2.2 \%$, and severe: $1.7 \%$ ) and also decreased to $6.0 \%$ at 1 month (mild: $3.3 \%$, moderate: $1.7 \%$, and severe: $1.1 \%$ ), $4.4 \%$ at 3 months (mild: $2.2 \%$, moderate: $1.1 \%$, and severe: $1.1 \%$ ), $2.2 \%$ at 6 months (mild: $0.6 \%$, moderate: $0.6 \%$, and severe, $1.1 \%$ ), and $1.7 \%$ over 12 months (mild: $0 \%$, moderate: $0.6 \%$, and severe: $1.1 \%$ )(Table 2 ).

Table 2

Comparison of the incidence and severity of dysphagia between AC and PC groups

\begin{tabular}{|llllllllll|}
\hline \multirow{2}{*}{ Timeframe } & None & \multicolumn{3}{c}{ Mild } & \multicolumn{2}{c|}{ Moderate } & Severe & p- \\
\cline { 2 - 9 } & AC & PC & AC & PC & AC & PC & AC & PC & \\
\hline Preoperative & $100.0 \%$ & $100.0 \%$ & $0.0 \%$ & $0.0 \%$ & $0.0 \%$ & $0.0 \%$ & $0.0 \%$ & $0.0 \%$ & \\
\hline $\begin{array}{l}\text { Early } \\
\text { postoperative }\end{array}$ & $87.21 \%$ & $90.65 \%$ & $5.81 \%$ & $5.49 \%$ & $4.07 \%$ & $2.20 \%$ & $2.91 \%$ & $1.65 \%$ & 0.806 \\
\hline 1 Month & $90.70 \%$ & $93.96 \%$ & $4.07 \%$ & $3.30 \%$ & $2.32 \%$ & $1.65 \%$ & $2.91 \%$ & $1.10 \%$ & 0.785 \\
\hline 3 Months & $93.02 \%$ & $95.60 \%$ & $2.32 \%$ & $2.20 \%$ & $1.74 \%$ & $1.10 \%$ & $2.91 \%$ & $1.10 \%$ & 0.801 \\
\hline 6 Months & $95.35 \%$ & $97.80 \%$ & $0.58 \%$ & $0.55 \%$ & $1.74 \%$ & $0.55 \%$ & $2.32 \%$ & $1.10 \%$ & 0.781 \\
\hline$>12$ Months & $96.51 \%$ & $98.35 \%$ & $0.58 \%$ & $0.0 \%$ & $1.74 \%$ & $0.55 \%$ & $1.16 \%$ & $1.10 \%$ & 0.749 \\
\hline
\end{tabular}

No significant difference was found between $A C$ and PC group in the incidence and severity of dysphagia early postoperatively or at any follow-up time (Table 2). We got totally different results compared with Smith-Hammond's report. ${ }^{2}$ 
Logistic regression model showed that usage time of neck collar after operation may lead to postoperative dysphagia. Age, gender, BMI, procedure type (AC versus PC), number of operated levels, operative time, revision surgery, and blood loss were not associated with an increased likelihood of dysphagia after AC and PC cervical procedure (Table 3).

Table 3

Results of Logistic Regression analysis.

\begin{tabular}{|llll|}
\hline Risk Factors & B & Sig. & Exp (B) \\
\hline Gender & 0.151 & 0.702 & 1.164 \\
\hline Age & 0.019 & 0.206 & 1.019 \\
\hline BMI & -0.022 & 0.682 & 0.979 \\
\hline operative levels & -0.434 & 0.445 & 0.648 \\
\hline Primary or revision & 0.292 & 0.434 & 1.339 \\
\hline Operative time & 0.216 & 0.797 & 1.241 \\
\hline Blood loss & 0.002 & 0.535 & 1.002 \\
\hline Usage time of neck collar after operation & 0.135 & 0.012 & 1.102 \\
\hline
\end{tabular}

\section{Discussion}

The occurrence of postoperative dysphagia after cervical spinal surgery in several studies ranged from from $4 \%-71 \%{ }^{1-25}$. In recent years, similar studies have been reported in China. These differences in the occurrence of dysphagia may be related to inclusion criteria, the design scheme and statistical method. In this study, the occurrence of dysphagia was $12.8 \%$ of patients in ACDF group and $9.4 \%$ in PC group. Dysphagia most commonly begins in the immediate postoperative period, but may also be delayed, beginning more than 4 weeks after surgery in some patients. Most cases are mild and resolve within 1 week. In a small subset of patients, these symptoms are permanent. Despite this, dysphagia has no significant impact on patient satisfaction with surgery.

A total of 354 patients were followed up by researcher in this study, including 172 patients of AC group and 182 patients of PC group. The results showed that dysphagia was $12.8 \%$ and $9.4 \%$ after AC and PC procedure in the early postoperative period. Symptoms then decreased to $9.3 \%$ and $6.0 \%$ at 1 month, $7.0 \%$ and $4.4 \%$ at 3 month, $4.6 \%$ and $2.2 \%$ at 6 months, 3.5\% and $1.6 \%$ at 12 months separately in the AC and PC group. No significant difference was found between AC and PC group in the incidence and severity of dysphagia early postoperatively or at any follow-up time after surgery. Since the incidence of postoperative dysphagia was nearly the same between these two groups, we hold the belief that the procedure type was not the key factor in the mechanism of developing dysphagia. Most of the 
improvement occurred in the first 3 months postoperatively. Most of the dysphagia was graded as "mild" and "moderate". Only $1.1 \%$ of patients at 1 year after cervical surgery had dysphagia that was graded as severe (Table 2).

Swallowing requires precise coordination of neuromuscular events in different organs, including oral, pharynx, laryngeal and esophagus ${ }^{10}$. The process of swallowing is divided into four parts: the first two named as oral pre-phase and oral phase, are voluntary movements involuntary dominated by human awareness; the last two named as throat phase and esophageal phase are involuntary movements. In cervical spinal surgery, Total or partial neuromuscular dysfunction during any stage of these parts may lead to the symptom of dysphagia after operation.

The mechanisms of postoperative dysphagia development after cervical spine surgery is currently under investigation. Masahiko Miyata ${ }^{3-4}$ and Neo reported that excessive change in 0-C2 angle at occipitalcervical fusion may result in reduction of the oropharyngeal volume, leading to severe dysphagia and even respiratory distress after operation. Michael-Johns ${ }^{5}$ considered postoperative dysphagia might be related to the throat phase that was divided into five segments, including epiglottis closure, retraction of the base of tongue, extrusion downwards of pharynx, closure and lift of laryngeal, and eventually relaxion of the upper esophageal sphincter. Cloward et al ${ }^{6}$ suggested that some degree of dysphagia is experienced by all patients after cervical operation.

To assess whether reported risk factors affected the occurrence of dysphagia, we collected clinical and operative characteristics. Logistic regression was performed in analyzing these clinical data, including gender, age, BMl, operation time, blood loss, type of surgical procedure (anterior/posterior), revision surgery, number of surgical segments, the superior segment of surgical cite, usage time of neck collar, whether the third cervical vertebrae was included. No direct association was observed between these data and postoperative dysphagia. But we find that long term use of neck collar may lead to postoperative dyaphagia(Table 3). That may partly because neck fixation for so long time is not conductive to eating and drinking. So we suggest that neck collar should be worn no longer than four weeks after cervical spine surgery.

The assessments of dysphagia in this retrospective study were based on the subjective experience by patients. Most patients presented with dysphagia after operation lack anatomical examinations such as barium meal test, and laryngoscopy ${ }^{18}$. In the future, prospective studies with larger samples are required to achieve more reliable conclusions. They can further provide guidance for surgical approach and lower the rate of postoperative complications.

\section{Conclusions:}

Dysphagia is a known complication after cervical spine surgery. The overall incidence of dysphagia was 12.8\% after $\mathrm{AC}$ and $9.4 \%$ after PC procedures in our study, and the incidence and severity both decreased over time. Long term use of neck collar after surgery is related of postoperative dysphagia. The neck 
collar should be worn no longer than four weeks after surgery. Age, gender, BMI, AC or PC procedures, operative time, blood loss, primary or revision and amount of levels operated on do not significantly increase the prevalence of dysphagia.

\section{Abbreviations}

1. The anterior cervical discectomy and fusion -- ACDF or AC

2. Posterior cervical spinous process-splitting laminoplasty --SLAC or PC

3. Body Mass Index--BMI

4. Statistical Product and Service Solutions--SPSS

5. Ossification of Posterior Longitudinal Ligament--OPLL

6. Arbeitsge-meinschaft für Osteosynthesefragen--AO

7. Computed Tomography--CT

\section{Declarations}

\section{Ethics approval and consent to participate}

The experimental protocol was established, according to the ethical guidelines of the Helsinki Declaration and was approved by the Human Ethics Committee of Beijing JISHUITAN Hospital. Written informed consent was obtained from individual or guardian participants.

\section{Consent to publication}

Not applicable.

\section{Availability of data and materials}

The datasets used or analysed during the current study are available from the corresponding author on reasonable request.

\section{Competing interests}

The authors have declared that no competing interests exist.

\section{Funding}

No Funding.

\section{Authors' Contributions}

JY and WT conceived and designed the study. JY performed the experiments and wrote the paper. WT reviewed and edited the manuscript. All authors read and approved the manuscript. 


\section{Acknowledgements}

We thank Shuo Liu, MD, MPH, for her contribution to the statistical analyses of this study. We also thank Peihao Jin, MD and Kai Yan, MD for their contributions as participating surgeons who enrolled patients in this study.

\section{References}

1. Bazaz R, Lee MJ, Yoo JU. Incidence of dysphagia after anterior cervical spine surgery: a prospective study. Spine (Phila Pa 1976). 2002; 27:2453-8.

2. Smith-Hammond CA, New KC, Pietrobon R, et al. Prospective analysis of incidence and risk factors of dysphagia in spine surgery patients: comparison of anterior cervical, posterior cervical, and lumbar procedures. Spine (Phila Pa 1976). 2004; 29:1441-6.

3. Miyata M, Neo M, Fujibayashi S, et al. O-C2 angle as a predictor of dyspnea and/or dysphagia after occipitocervical fusion. Spine (Phila Pa 1976). 2009; 34: 184-8.

4. Yoshida M, Neo M, Fujibayashi S, et al. Upper-airway obstruction after short posterior occipitocervical fusion in a flexed position. Spine (Phila Pa 1976). 2007; 32:E267-70.

5. Michael M, Johns III, Thomas E. Dysphagia Following Anterior Cervical Spine Surgery. Seminars in Spine Surgery. 2004; 10:274-278.

6. Cloward RB. New method of diagnosis and treatment of cervical disc disease. Clin Neurosurg 1962; 8: 93-127.

7. Varun Puvanesarajah, Amit Jain, Adam L. Shimer. Complications and Mortality Following One to Two-Level Anterior Cervical Fusion for Cervical Spondylosis in Patients Above 80 Years of Age. Spine (Phila Pa 1976) 2017;42:E509-E514.

8. Shuichi Kaneyama, Masatoshi Sumi, Masato Takabatake. The Prediction and Prevention of Dysphagia After Occipitospinal Fusion by Use of the S-line (Swallowing Line). Spine (Phila Pa 1976) 2017;42:718-725.

9. Jeremie D. Oliver, Sandy Goncalves, Panagiotis Kerezoudis. Comparison of Outcomes for Anterior Cervical Discectomy and Fusion With and Without Anterior Plate Fixation.Spine (Phila Pa 1976);2018;43:E413-E422.

10. Bryce A. Basques, Fady Y. Hijji, Benjamin Khechen. Sex Differences for Anterior Cervical Fusion-Complications and Length of Stay. Spine (Phila Pa 1976) 2018;43:1025-1030.

11. Peter B. Derman, Lukas P. Lampe, Ting Jung Pan. Postoperative Emergency Department Utilization and Hospital Readmission After Cervical Spine Arthrodesis. Spine (Phila Pa 1976) 2018;43:10311037.

12. Stephen Clark, Alysa Nash, Mark Shasti. Mortality Rates After Posterior C1-2 Fusion for Displaced Type II Odontoid Fractures in Octogenarians. Spine (Phila Pa 1976) 2018;43:E1077-E1081. 
13. Andrew Y. Yew, Matthew T. Nguyen, Wellington K. Hsu. Quantitative Risk Factor Analysis of Postoperative Dysphagia After Anterior Cervical Discectomy and Fusion (ACDF) Using the Eating Assessment Tool-10 (EAT-10). Spine (Phila Pa 1976) 2019;44:E82-E88.

14. Ichiro Kawamura, Hiroyuki Tominaga, Fumito Tanabe. Cervical Alignment of Anterior Cervical Hyperostosis Causing Dysphagia. Spine (Phila Pa 1976) 2019;44:E269-E272.

15. Avani S. Vaishnav, Philip Saville, Steven McAnany. Predictive Factors of Postoperative Dysphagia in Single-Level Anterior Cervical Discectomy and Fusion. Spine (Phila Pa 1976) 2019;44:E400-E407.

16. Jae Taek Hong, Joo Seon Oh, Dong Hoon Lee. Association Between the Severity of Dysphagia and Various Parameters of the Cervical Spine; Videofluoroscopic Analysis in Neutral and Retraction Position of the Normal Volunteers. Spine (Phila Pa 1976) 2020;45:103-108.

17. Comparison of anterior controllable antedisplacement and fusion (ACAF) with posterior laminoplasty in the treatment of multilevel cervical ossification of the posterior longitudinal ligament: $A$ prospective, randomized and control study with at least one-year follow up. Spine (Phila Pa 1976) 2020 Publish Ahead of Print. DOI: 10.1097/BRS.0000000000003462.

18. Shari Cui, MD, Scott D. Daffner, MD, John C. France, MDThe Effects of Perioperative Corticosteroids on Dysphagia Following Surgical Procedures Involving the Anterior Cervical Spine--A Prospective, Randomized, Controlled, Double-Blinded Clinical Trial. J Bone Joint Surg Am. 2019;101:2007-14.

19. Alexander P. Hughes, Stephan N. Salzmann, Okezie K. Aguwa. HSS Dysphagia and Dysphonia Inventory (HSS-DDI) Following Anterior Cervical Fusion--Patient-Derived, Validated, ConditionSpecific Patient-Reported Outcome Measure Outperforms Existing Indices. J Bone Joint Surg Am. 2018;100:e66(1-10).

20. Tyler James Jenkins, Rueben Nair, Surabhi Bhatt. The Effect of Local Versus Intravenous Corticosteroids on the Likelihood of Dysphagia and Dysphonia Following Anterior Cervical Discectomy and Fusion--A Single-Blinded, Prospective, Randomized Controlled Trial.

21. Narihito Nagoshi, Lindsay Tetreault, Hiroaki Nakashima. Risk Factors for and Clinical Outcomes of Dysphagia After Anterior Cervical Surgery for Degenerative Cervical Myelopathy--Results from the AOSpine International and North America Studies. J Bone Joint Surg Am. 2017;99:1069-77.

22. Yang Meng, Tingkui Wu, Ziyang Liu. The impact of the difference in O-C2 angle in the development of dysphagia after occipitocervical fusion: a simulation study in normal volunteers combined with a case-control study. The Spine Journal 18 (2018) 1388-1397.

23. Lin-nan Wang, Bo-wen Hu, Yue-ming Song. Predictive abilities of O-C2a and O-EAa for the development of postoperative dysphagia in patients undergoing occipitocervical fusion. The Spine Journal 20 (2020) 745-

24. Seok Woo Kim, Chulyoung Jang, Myung Ho Yang. The natural course of prevertebral soft tissue swelling after anterior cervical spine surgery: how long will it last? The Spine Journal 17 (2017) 1297-1309.

25. Avani S. Vaishnav, Philip Saville, Steven McAnany. Is the likelihood of dysphagia different in patients undergoing one-level versus two-level anterior cervical discectomy and fusion? The Spine Journal 20 


\section{Tables}

Table 1. Bazaz grading system for dysphagia

\begin{tabular}{|lllr|}
\hline Severity & Liquid & Solid & \\
\hline None & None & None & \\
\hline Mild & None & Rare & (only with specific foods) \\
Moderate & None or Rare & Occasional & (majority of Solids) \\
\hline Severe & Present & Frequent & (2) \\
\hline
\end{tabular}

Table 2.Comparison of the incidence and severity of dysphagia between AC and PC groups

\begin{tabular}{|llllllllll|}
\hline \multirow{2}{*}{ Timeframe } & None & & \multicolumn{2}{c}{ Mild } & \multicolumn{2}{c|}{ Moderate } & \multicolumn{2}{l|}{ Severe } & $\begin{array}{c}\text { p- } \\
\text { value }\end{array}$ \\
\cline { 2 - 10 } & AC & PC & AC & PC & AC & PC & AC & PC & \\
\hline Preoperative & $100.0 \%$ & $100.0 \%$ & $0.0 \%$ & $0.0 \%$ & $0.0 \%$ & $0.0 \%$ & $0.0 \%$ & $0.0 \%$ & \\
\hline $\begin{array}{l}\text { Early } \\
\text { postoperative }\end{array}$ & $87.21 \%$ & $90.65 \%$ & $5.81 \%$ & $5.49 \%$ & $4.07 \%$ & $2.20 \%$ & $2.91 \%$ & $1.65 \%$ & 0.806 \\
\hline 1 Month & $90.70 \%$ & $93.96 \%$ & $4.07 \%$ & $3.30 \%$ & $2.32 \%$ & $1.65 \%$ & $2.91 \%$ & $1.10 \%$ & 0.785 \\
\hline 3 Months & $93.02 \%$ & $95.60 \%$ & $2.32 \%$ & $2.20 \%$ & $1.74 \%$ & $1.10 \%$ & $2.91 \%$ & $1.10 \%$ & 0.801 \\
\hline 6 Months & $95.35 \%$ & $97.80 \%$ & $0.58 \%$ & $0.55 \%$ & $1.74 \%$ & $0.55 \%$ & $2.32 \%$ & $1.10 \%$ & 0.781 \\
\hline$>12$ Months & $96.51 \%$ & $98.35 \%$ & $0.58 \%$ & $0.0 \%$ & $1.74 \%$ & $0.55 \%$ & $1.16 \%$ & $1.10 \%$ & 0.749 \\
\hline
\end{tabular}

Table 3. Results of Logistic Regression analysis. 


\begin{tabular}{|lcll|}
\hline Risk Factors & B & Sig. & Exp (B) \\
\hline Gender & 0.151 & 0.702 & 1.164 \\
\hline Age & 0.019 & 0.206 & 1.019 \\
\hline BMI & -0.022 & 0.682 & 0.979 \\
\hline Procedure type (AC versus PC) & -0.434 & 0.445 & 0.648 \\
\hline operative levels & 0.292 & 0.434 & 1.339 \\
\hline Primary or revision & 0.216 & 0.797 & 1.241 \\
\hline Operative time & 0.002 & 0.535 & 1.002 \\
\hline Blood loss & 0.000 & 0.558 & 0.999 \\
\hline Usage time of neck collar after operation & 0.135 & 0.012 & 1.102 \\
\hline
\end{tabular}

Figures 


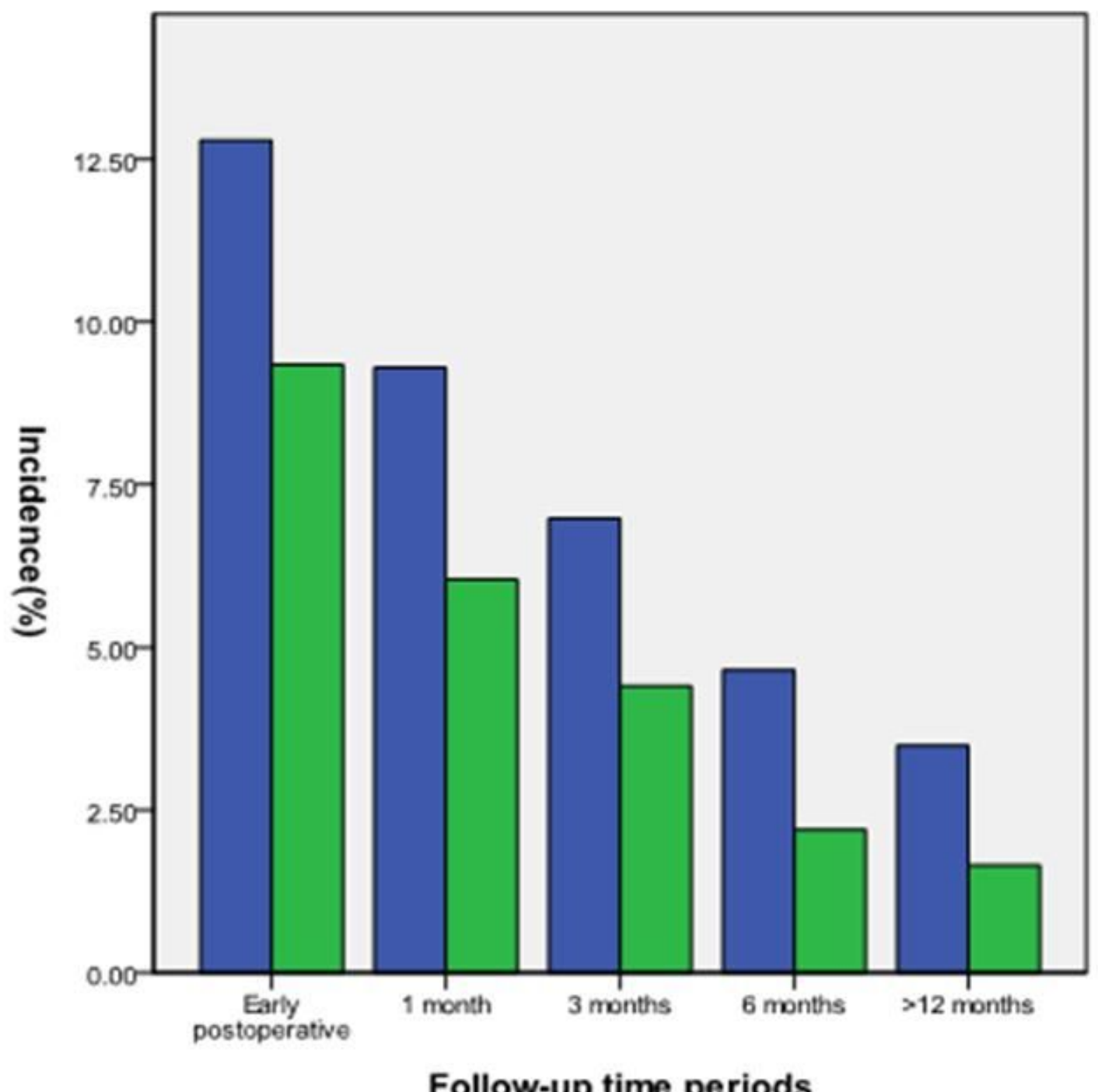

Procedure type

$\square \mathrm{AC}$

$\square P C$

Follow-up time periods

Figure 1

Comparison of the overall incidence of dysphagia between the AC and PC group early preoperatively and at each time period after surgery (early postoperative, 1 month, 3 months, 6 months, and 12 months). 


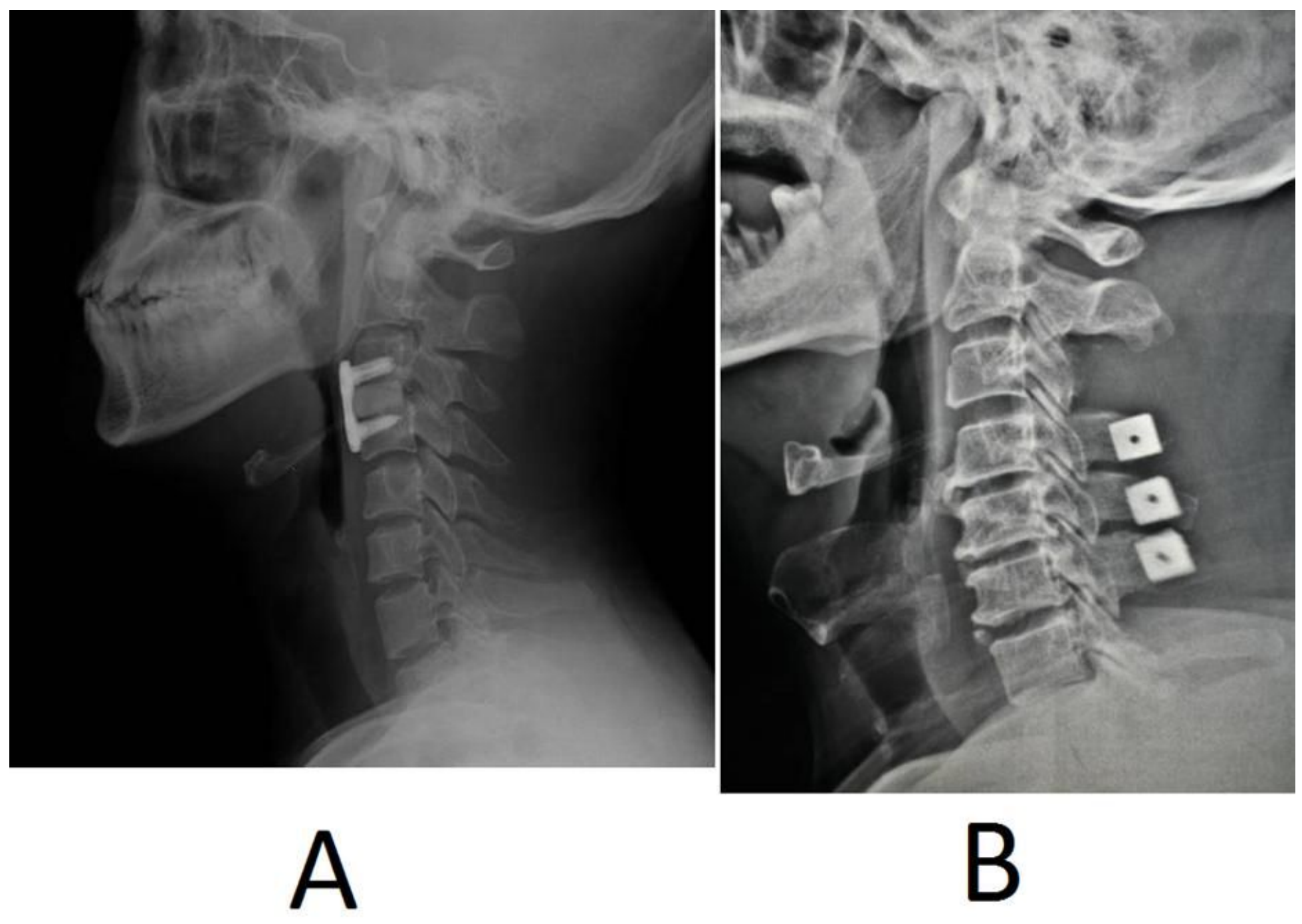

Figure 2

X-ray lateral cervical photograph of patients following ACDF/posterior cervical spinous process-splitting laminoplasty. F 2-a. photograph of patient following ACDF F 2-b. photograph of patients following cervical spinous process-splitting laminoplasty. 